\title{
Dideoxyfingerprinting (ddF) Analysis of the Type X Collagen Gene (COL10A1) and Identification of a Novel Mutation (S671P) in a Kindred with Schmid Metaphyseal Chondrodysplasia
}

\author{
Constantine A. Stratakis,*,, 1 Zsolt Orban, $¥$ A. Lee Burns, $¥$ Alessandra Vottero,* \\ Constantine S. Mitsiades,* Stephen J. Marx, $¥$ Val Abbassi,† and George P. Chrousos*
}

\begin{abstract}
*Section on Pediatric Endocrinology, Developmental Endocrinology Branch, National Institute of Child Health and Human Development (NICHD) and ¥Genetics and Endocrinology Section, Metabolic Diseases Branch, National Institute of Diabetes, Digestive and Kidney Diseases (NIDDK), National Institutes of Health (NIH), Bethesda, Maryland 20892; and †Division of Pediatric

Endocrinology, Department of Pediatrics, Georgetown University Children's Medical Center, Washington, DC 20007
\end{abstract}

Received J uly 9, 1996; and in revised form August 20, 1996

Schmid metaphyseal chondrodysplasia (SMCD; MIM 156500) is an autosomal dominant disorder of the skeleton that is manifested in early childhood by short stature, coxa vara, and a waddling gait. Patients with SMCD have mutations in the gene that codes for the $\alpha-1$ chain of collagen X (COL10A1); however, mutation analysis of this gene is hampered by its size. We studied a family with SMCD: the mother, a 36-year-old woman with a height of $149 \mathrm{~cm}$, had mild bilateral coxa vara. Her two sons presented with short stature, bowed legs, and coxa vara in early childhood. DNA was extracted from peripheral lymphocytes from the three patients and subjected to PCR amplification by COL10A1 genespecific primers. In addition to single-strand conformational polymorphism (SSCP) analysis of the COL1OA1 gene, we used a novel method, dideoxy fingerprinting (ddF). The genetic defect in this family was found to be a previously unreported missense mutation (T-to-C transition) at nucleotide 2011. This change resulted in a Ser-to-Pro substitution at position 671 of the carboxy-terminus of the COL 10A1 protein. In addition, the two boys, but not the mother, were found to carry a trinucleotide (CCC) deletion at position 2048 of the 3' untrans-

\footnotetext{
${ }^{1}$ To whom correspondence and reprint requests should be addressed at Section on Pediatric Endocrinology (SPE), Developmental Endocrinology Branch (DEB), National Institute of Child Health \& Human Development (NICHD), National Institutes of Health (NIH), 9000 Rockville Pike, Building 10, Room 10 N 262, Bethesda, MD 20892-1862. Fax: (301) 402-0574. E-mail: stratakc@ccl.nichd.nih.gov.
}

lated region, a polymorphism of the COL 10A1 gene. We conclude that ddF can be used in the analysis of the COL 1OA1 gene along with SSCP. The S671P substitution is novel, but located in the same region with the other reported COL 10A1 mutations, confirming type $X$ collagen as the locus for this disease.

Schmid metaphyseal chondrodysplasia (SMCD; MIM 156500) is a rare, autosomal dominant disorder of the skeleton that is manifested in early childhood by short stature, coxa vara, and a waddling gait (1). Mutations in the second exon of the gene that codes for the $\alpha$-1 chain of collagen X (COL 10A1) were recently identified in patients with this condition (28). Collagen $X$ is a member of the family of "short chain" collagen molecules, a unique component of the extracellular matrix essential for normal growth plate function (9). It is expressed in bone development transiently and its message is confined to the terminally differentiated hypertrophic chondrocytes in the calcifying cartilage of the growth plate. Its function is not completely understood, but it has been suggested that this molecule provides the structural support necessary for vascular invasion, calcification, and remodeling of the growing bone $(9,10)$. The protein has a triple-helical (COL) domain of 463 amino acid residues, flanked by two nontriple helical sequence domains, one at the carboxyl-terminus (NC1), and another at the amino-terminus (NC2) of the molecule $(10,11)$. The 161-amino-acid 

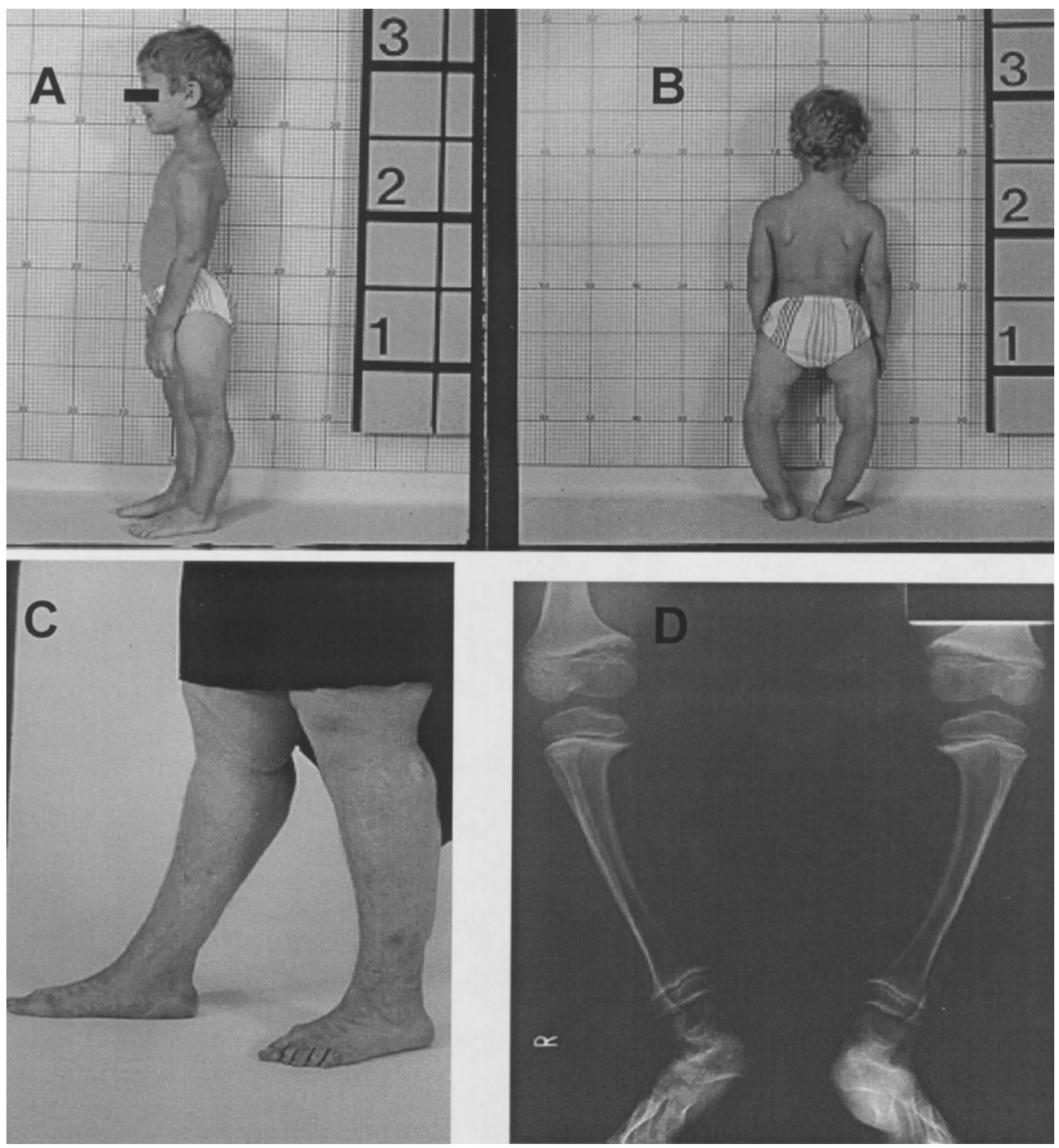

FIG. 1. Patients' phenotypes. A, B, and C, are from individuals II.1, II.2, and I.1, respectively. Patient I.1 underwent two operations, first as a child, and then as a teenager; neverthless, her leg bowing was never as severe as that of her two sons. (D) Radiograph of the lower extremities of patient II.2: significant bowing of the tibiae.

$\mathrm{NCl}$ domain is highly conserved among species and is bel ieved to be required for the specific associations that occur during assembly of the triple helix (11). Both the NC1 and the NC2 domains are the sites of extensive posttranslational modification by specific $\mathrm{C}$ - and $\mathrm{N}$-terminal proteinases, respectively, as well as by lysyl oxidase (10-12).

The three $\alpha-1$ (X) chains are encoded by a single gene, which is located on the distal end of the long arm of chromosome 6 (6q21- q22.3) and has two exons; exon 2 is 2940 bp long and codes for over $95 \%$ of the $\alpha-1(\mathrm{X})$ chain, including the $\mathrm{COL}$ and $\mathrm{NCl}$ domains and the $3^{\prime}$-untranslated sequence $(11,12)$. Because of its time and growth-plate-restricted expression and function, COL 10A1 was the candidate gene for the chondrodysplasias, which affect primarily the developing growth plate. Over the past 2 years, 12 mutations have been described in kindreds with SMCD, all concentrated in the $\mathrm{NCl}$ domain of the protein. The first mutation described was a 13bp del etion leading to a truncated COL $10 A 1$ protein (2); 6 deletions and 5 missense mutations of this gene have been reported since $(3,5,6-8)$. The screening of COL 10A1 for mutations is hampered by the 
size of its second and largest exon. Although singlestrand conformational polymorphism (SSCP) analysis was successfully employed for identification of new mutations in the past $(3,5,6,14)$, in at least one case this technique failed to identify a missense mutation, which was subsequently identified by heteroduplex analysis (3). In this study, we report the use of a novel method for screening mutations of the COL 10A1 gene, dideoxy fingerprinting (ddF), and describe a novel mutation in the $\mathrm{NCl}$ domain of the protein, which caused the disease in a Ukrainian kindred.

\section{MATERIALS AND METHODS}

\section{Family Report}

The family came to the United States for medical evaluation and treatment. They were previously given the diagnosis of " $X$-linked hypophosphatemic rickets" (XLHPR). The mother (patient I.1) was 36 years old and as a child had mild coxa vara and bowed legs, which came to medical attention at the age of 5 years. She underwent osteotomy twice; she completed a normal puberty at the age of 15 and had no other medical problems. Her family history was negative for any skeletal disorders, and her diagnosis was "rickets" for which she received occasional treatment with vitamin D and calcium supplements. At the ages of 30 and 31 years, she gave birth to male children II.1 and II.2, respectively, who were delivered vaginally and had normal birth length and weight. They both had normal early development, but when they started walking, shortly after the end of the first year of life, they developed significant leg bowing. Although their original diagnosis was hypochondroplasia, the two brothers and their mother were subsequently diagnosed with XLHPR. At their physical examination in our institution, the brothers had short stature due to bilaterally bowed legs (Fig. 1) with a short lower body segment and a normal upper body segment; arm span length was normal for age. Both boys walked with a waddling gait and had a small thorax and genu varum. There were no other abnormal findings. The mother (I.1) was also short (height $149 \mathrm{~cm},-2.5 \mathrm{SD}$ ); she had numerous scars in her lower extremities from the corrective osteotomies, but was able to walk normally. Radiographic evaluation of the three patients, showed findings typical of SMCD without any evidence for rickets. Coxa vara was present in both boys, but not in their mother. The femoral capital
TABLE 1

\section{Primers Used for SSCP, ddF, and Sequence Analyses of the COL1OA1 Gene ${ }^{a}$}

\begin{tabular}{lll}
\hline Primer & \multicolumn{1}{c}{ Sequence } & \multicolumn{1}{c}{$\begin{array}{c}\text { cDNA location } \\
\text { (Ref. 12) }\end{array}$} \\
\hline X16F & 5'-CACCTGCATGTGAAAGGG-3' & 1804 (Sense) \\
X16R & 5'-GGGTGGGGTAGAGTTAGA-3' & 2115 (Antisense) \\
X22F & 5'-GCCTGTATAAGAATGGCACC-3' $^{\prime}$ & 1838 (Sense) \\
CXFo & 5'-GCAACAGCATTATGACCCAAGG-3' & 1749 (Sense) \\
CXRe & 5'-TACATTCTTTTCAGCCTACCTCC-3' & 2160 (Antisense) \\
\hline
\end{tabular}

${ }^{\text {a }}$ For additional primers see also Ref. (14).

epiphysis was enlarged and malformed in both children; the spine and other parts of the skeleton were normal. Biochemical investigation revealed normal serum and urine cal cium and phosphorus concentrations. Serum parathyroid hormone (PTH), vitamin $D$ metabolites, and alkaline phosphatase levels were also normal.

\section{Methods}

Genomic DNA was prepared from the patients and normal controls as previously described (13). PCR amplification, SSCP, and ddF analyses were performed with primers previously reported $(2,3,5,14)$. Simple PCRs were first performed in order to detect major gene deletions or rearrangements. The products of these reactions were run on ethidium bromide-stained $1 \%$ agarose gels and were found to be of the expected size (data not shown). The conditions of the PCR and SSCP analysis were previously described $(3,5,14)$. For the new primers (Table 1$)$, the conditions were slightly different: the reactions were carried in a $100-\mu$ l volume containing $\sim 100 \mathrm{ng}$ of DNA, 10 pmol of each unlabeled primer, $20 \mathrm{nmol}$ of each dNTP, in $1.5 \mathrm{~mm} \mathrm{MgCl}$ PCR buffer, with $1 \mathrm{U}$ of Taq polymerase (Perkin-Elmer, Norwalk, CT). Thirty cycles were performed $\left(94^{\circ} \mathrm{C}\right.$ for $1 \mathrm{~min}, 57^{\circ} \mathrm{C}$ for $1 \mathrm{~min}, 72^{\circ} \mathrm{C}$ for $30 \mathrm{~s}$ ) followed by a final 10 -min extension at $72^{\circ} \mathrm{C}$. ddF analysis was performed as described (15). Primers CXFo and CXRe were used for generation of an amplicon encompassing the NC1 domain-coding sequence of the COL 10Al gene (nucleotides 1749-2160 of the CDNA), with conditions similar to the ones described above. A nested T-4 kinase end-labeled primer (13) was then used for the $\mathrm{ddF}$ reaction. For a total volume of $10 \mu \mathrm{l}$, the reaction

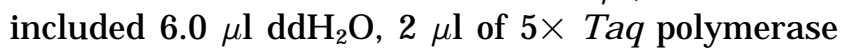
buffer (Invitrogen, San Diego, CA), $0.1 \mu \mathrm{l}$ of $2.5 \mathrm{~mm}$ 
NTP, $0.2 \mu \mathrm{l}$ of $10 \mathrm{~mm}$ ddGTP/L, $2 \mathrm{pmol} / \mathrm{liter}(1 \mu \mathrm{l})$ of labeled primer, $0.2 \mu \mathrm{l}(5 \mathrm{U} / \mu \mathrm{l})$ Taq polymerase (Perkin-Elmer, Norwalk, CT), and $0.5 \mu$ l of the amplicon prepared above. Forty cycles of PCR were then performed and stopped with the addition of $15 \mu \mathrm{l}$ of stop solution ( $7 \mathrm{~mol} / \mathrm{liter}$ urea, $50 \%$ formamide, $3 \mathrm{mmol} /$ liter EDTA, $0.5 \%$ bromphenol-blue-xylene cyanol). Three to five microliters was loaded in a standard $1 \times$ MDE nondenaturing gel (AT Biochem, Malvern, PA), after heating the samples to $95^{\circ} \mathrm{C}$ for $5 \mathrm{~min}$. The gel was run at constant power for $8 \mathrm{~h}$, dried, and subjected to autoradiography. Following the identification of the change by SSCP and ddF, 10 normal control individuals were screened by SSCP for mutations in the $\mathrm{NCl}$ domain of the COL 10A1 gene and a normal pattern was revealed (data not shown). cDNA synthesis was accomplished from total RNA extracted from the EBV-transformed lymphocytes of the patients and two normal control cell lines as previously reported (16). The reverse transcriptase-PCR kit (Boehringer-Mannheim, Mannheim, Germany) was used for this reaction. Primer X16R at position 2115 of the $3^{\prime}$-untranslated sequence was used for reverse transcription and the previously used 5'E (X22F) for subsequent amplification by PCR. Direct and asymmetric PCR sequencing was performed as we previously reported (16) by the fmol-sequencing method (Promega Corp., Madison, MI), and the Sequenase version 2.0 kit (U.S. Biochemicals, Cleveland, $\mathrm{OH}$ ), respectively.

\section{RESULTS}

PCR analysis of 5 fragments of the COL 10A1 gene in the 3 patients and 1 normal control revealed amplicons of the expected size, excluding major deletions or other major rearrangements of this gene (data not shown). SSCP analysis of the COL10A1 gene in the 3 patients and 3 controls was then performed for 10 amplicons covering the full length of the COL 10A1 coding sequence. SSCP alterations were found in amplicon $E$ (primers $X 22 F$ and $X 22 R$ ), which covers the distal 271 bp of exon 2 of the COL10A1 gene and includes parts of the NCl domain-coding sequence and the 3 '-end untranslated sequence (Fig. 2). However, the band pattern of the two affected children was different from that of their mother, although all three affected individuals had bands which were different from those of the normal controls. ddF analysis showed that, indeed, there was variance between the band patterns of the mother and the two affected children, but not for

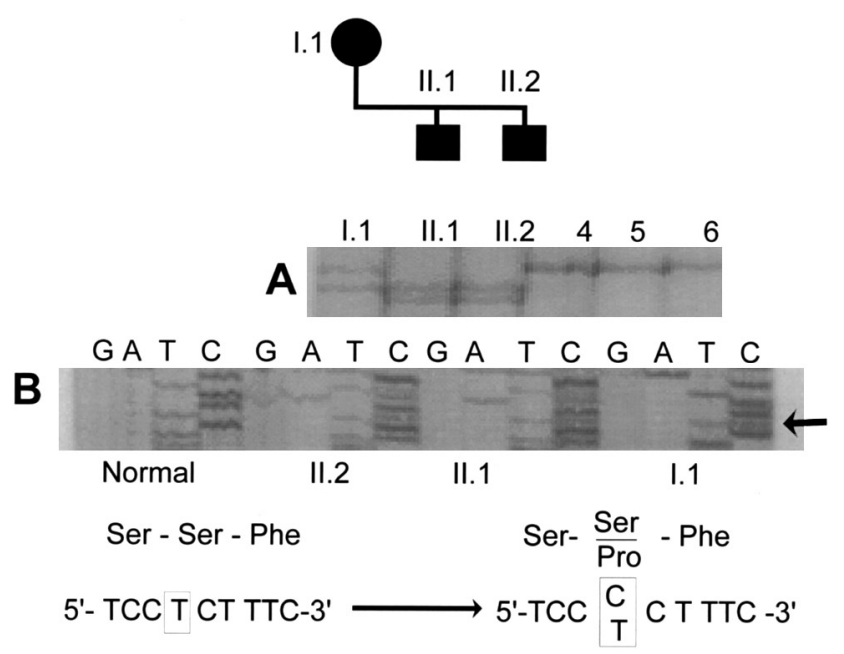

FIG. 2. SSCP and sequence analysis of the COL $10 A 1$ genes. (A) SSCP analysis of the COL 10A1 gene [NC-1 domain, amplicon E (Ref. 14)] from the mother (I.1), her two sons (II.1 and II.2) and three normal controls. (B) Sequencing results indicating the T/ C missense mutation at position 2011 of the COL10A1 coding sequence and the amino acid substitution S671P (the gel reads from top to bottom in the 5'-to-3' direction). In the two children there is an overlap in the sequence, due to the CCC $(-)$ polymorphism (see Figs. 3 and 4).

the entire length of the amplicon (Fig. 3). No other changes were detected by SSCP and/or ddF analysis of the rest of the COL1OA1-coding and proximal intronic sequences.

The COL 10A1 CDNA obtained by reverse transcription of total RNA from EBV-transformed lymphocytes was of the expected length, excluding splice-site mutations or other genetic defects that would alter the transcription of the COL10A1 gene (data not shown). Sequencing of amplicon $E$ revealed two sequence alterations: the first was present in all three patients; the second was present in the boys only. The former was a missense mutation at nucleotide position 2011 of the COL 10A1 coding sequence, which replaced a thymidine $(T)$ with a cytosine (C) in the first base of a TCT codon. This mutation led to a nonconservative amino acid substitution at position 671 of the $\mathrm{NC1}$ domain of the protein, changing serine (Ser, S) to proline (Pro, P) (5671P) (Fig. 2). The second sequence alteration, present only in the two boys and inherited apparently from their unaffected father, was the CCC $(-)$ polymorphism of the COL 10A1 gene, which is located 6 bp downstream from the TGA termination codon and results in a 3bp deletion (CCC) at position 2048 of the COL 10A1 CDNA (12) (Fig. 4). This polymorphism was present 


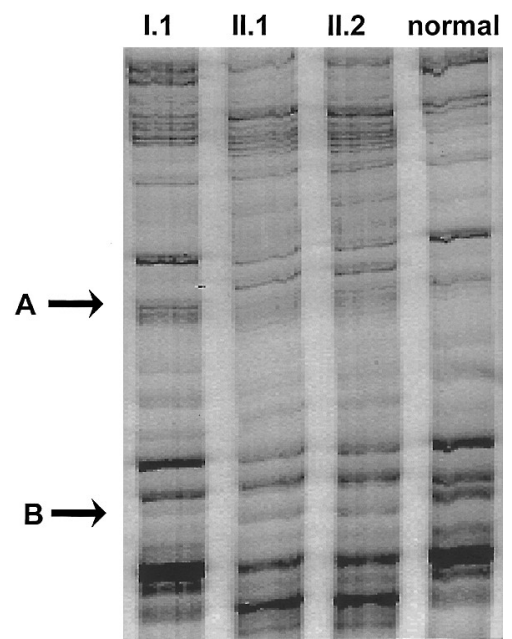

FIG. 3. Dideoxy fingerprinting (ddF) analysis of an amplicon of the COL 10A1 exon 2, encompassing the same region as that studied by SSCP (Fig. 2A) with an antisense primer. From the top of the gel to arrow $A$, all lanes have a band pattern which is similar in the mother (I.1) and her two children (II.1 and II.2) and different from that of the control, as a result of the S671P mutation. As we approach sequences that are closer to the primer (after arrow B) the band pattern differs between the mother and her two children because of the $\mathrm{CCC}(-)$ deletion, a polymorphism of the COL $10 A 1$ gene.

in 1 of the 10 normal control individuals screened by SSCP analysis (data not shown) and was responsible for the difference in the band patterns between the mother and her two affected children, as detected by SSCP and ddF analysis.

\section{DISCUSSION}

Several lines of evidence suggest that the S671P mutation is responsible for SMCD in our kindred. First, the mutation segregated with the disease in all three affected individuals. Second, this mutation was located in the NC-1 domain of the COL $10 \mathrm{~A} 1$ gene product, where 12 other, different mutations have been identified in patients with SMCD. Thus, we suggest that its role in the pathogenesis of this disorder is similar to the one proposed for the other disease-causing mutations, i.e., disruption of trimer assembly of the collagen $X$ molecule. Third, as observed for the sites of the other reported mutations, the serine moiety at position 671 is conserved in sequences of this molecule from all the species published to date, including the avian, murine, and bovine genes $(11,12)$. Finally, the nature of the amino acid change indicates that this mutation may directly affect the function of the collagen $X$ molecule.
Substitution of a serine by a proline may offer an additional sitefor proteolysis; in addition, serine residues favor the formation of reverse turns in the $\alpha$ helix structure of polypeptide chains, whereas proline moieties generally disrupt the $\alpha$-helix formation. The end result of these modifications would be either haploinsufficiency of the COL 10A1 gene product, or a dominant-negative effect in the structure of the collagen $X$ homotrimer, or both.

An in vitro transcription system was recently used in order to show that substitution of glycine at position 618 of the human COL 10A1 gene by valine disturbed the aggregation of collagen molecules into trimers and larger assemblies (8). This finding was consistent with the abnormal disorganized cartilage seen in the growth plate of the patient with this mutation, although it did not provide any details for the mechanism by which this abnormality occurred. Transfection studies with wild-type and mutant COL 10A 1 sequences have not been reported and direct proof of the involvement of the reported mutations in disease pathogenesis is currently lacking. It is noteworthy that homozygous murine knockouts for type $X$ collagen have a normal phenotype, indicating species-specific differences in the function of this molecule $(9,10)$.

The S671P substitution is the mutation closest to the TGA-stop codon identified to date, extending the region of disease-causing substitutions al most to the end of the coding sequence of the COL1OAl gene. Definite phenotype- genotype correlations are lacking, although there do not appear to be significant differences in the clinical severity of the disease between patients bearing deletions and those who are carriers of missense mutations. It is possible that the observed wide variation in clinical symptomatol-

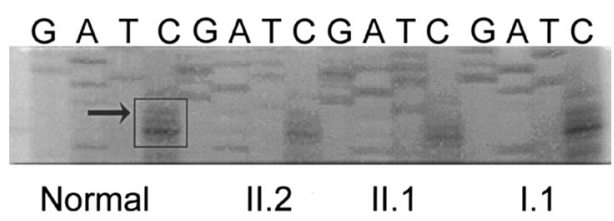

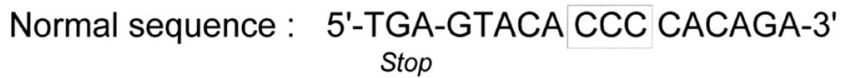
CCC(-) polymorphism : 5'-TGA-GTACACACAGA-3'

FIG. 4. Sequencing ladder indicating the overlap in nucleotides due to the $\mathrm{CCC}(-)$ polymorphism in the paternal COL $10 \mathrm{~A} 1$ allele in the two children (II.1 and II.2) (the CCC trinucleotide is shown in the box). The mother (I.1) has the same sequence as the normal control individual (four leftmost col umns). The normal and polymorphic sequences are shown at the bottom. 
ogy of patients with SMCD is due to other factors, perhaps the function of other genes. The possibility that polymorphisms of the COL 10A1 gene might play a role in disease expression has not been investigated. In particular, a polymorphism of the proximal 3'-untranslated sequence of the COL 10A1 coding sequence, for which the carrier frequency is close to $10 \%$ in at least one studied population (14), may play a role in COL 10A1 mRNA stability because of its position (17). This polymorphism, a trinucleotide (CCC) deletion at position 2048 of the CDNA, was present in the two children but not in their mother. It is tempting to speculate that the unusual severity of SMCD in the two children (bowing of the extremities before the age of 2 years and severe damage of the femoral capital epiphysis) was due to the fact that they were carrying not only the disease-causing mutation, in the maternal allele, but also the trinucleotide deletion, which may have reduced the stability of the message produced from the paternally inherited COL 10A1 allele. The presence of this polymorphism in unaffected individuals (14) does not exclude this possibility, since such an effect would only be clinically significant in cases where any amount of normal collagen $X$ formation would be critical. This suggests that further studies are needed in order to obtain a better understanding of the effect of polymorphisms of the COL 10A1 gene on the phenotype of SMCD patients.

\section{REFERENCES}

1. Lanchman RS, Rimoin DL, Spranger J. Metaphyseal chondrodysplasia, Schmid type: Clinical and radiographic delineation with a review of the literature. Pediatr Radiol 18: 93- 102, 1988.

2. Warman $M L$, Abbott $A$, Apte SS, Hefferon $T, M c l$ ntosh I, Cohn DH, Hecht J T, Olsen BR, Francomano CA. A type X collagen mutation causes Schmid metaphyseal chondrodysplasia. Nature Genet 5: 79- 82, 1993.

3. Mclntosh I, Abbott MH, Warman ML, Olsen BR, Francomano $C A$. Additional mutations of type $X$ collagen confirm COL 10A1 as the Schmid metaphyseal chondrodysplasia locus. Hum Mol Genet 3: 303-307, 1994.

4. Dharmavaram RM, Elberson MA, Peng M, Kirson LA, Kelley $T E$, J imenez $S A$. Identification of a mutation in type $X$ collagen in a family with Schmid metaphyseal chondrodysplasia. Hum Mol Genet 3: 507-509, 1994.

5. Wallis GA, Rash B, Sweetman WA, Thomas J T, Super M,
Evans G, Grant ME, Boot-Handford RP. Amino acid substitutions of conserved residues in the carboxyl-terminal domain of the al $(X)$ chain of type $X$ collagen occur in two unrelated families with metaphyseal chondrodysplasia type Schmid. Am J Hum Genet 54: 169- 178, 1994.

6. Mclntosh I, Abbott MH, Francomano CA. Concentration of mutations causing Schmid metaphyseal chondrodysplasia in the C-terminal non-collagenous domain of type-X collagen. Hum Mutat 5: 121- 125, 1995.

7. Pokharel RK, Alimsardjono H, Uno K, Fuji S, Shiba R, Matsuo M. A novel mutation substituting tryptophan with arginine in the carboxyl-terminal, non-collagenous domain of collagen $X$ in a case of Schmid metaphyseal chondrodysplasia. Biochem Biophys Res Commun 217: 1157-1162, 1995.

8. Chan D, Cole WG, Rogers J G, Baterman J F. TypeX collagen multimer assembly in vitro is prevented by a Gly ${ }^{618}$ to Val mutation in the al(X) NCl domain resulting in Schmid metaphyseal chondrodysplasia. J Biol Chem 270: 4558-4562, 1995.

9. Kivirikko KI. Collagens and their abnormalities in a wide spectrum of diseases. Ann Med 25: 113-126, 1993.

10. Olsen BR. Mutations in collagen genes resulting in metaphyseal and epiphyseal dysplasias. Bone 17: 45S-49S, 1995.

11. Apte SS, Seldin MF, Hayashi M, Olsen BR. Cloning of the human and mouse type $X$ collagen genes and mapping of the mouse type $X$ collagen gene to chromosome 10. Eur J Biochem 206: 217-224, 1992.

12. Thomas J T, Creswell CJ, Rash B, Nicolai H, J ones T, Solomon E, Grant ME, Boot-Handford RP. The human collagen $X$ gene: Complete primary translated sequence and chromosomal localization. Biochem J 280: 617-623, 1991.

13. Stratakis CA, Carney J A, Lin J P, Papanicolaou DP, Karl M, Kastner DL, Pras E, Chrousos GP. Carney complex, a familial multiple neoplasia and lentiginosis syndrome: Analysis of 11 kindreds and linkage to the short arm of chromosome 2. J Clin Invest 97: 599-607, 1996.

14. Sweetman WA, Rash B, Beighton P, Hecht JT, Zabel B, Thomas J T. SSCP and segregation analysis of the human type $X$ collagen gene (COL 10A1) in heritable forms of chondrodysplasia. Am J Hum Genet 51, 841-849, 1992.

15. Felmlee TA, Liu Q, Whelen AC, Williams D, Sommer SS, Persing DH. Genotyping detection of Mycobactreium tuberculosis rifampin resistance: Comparison of single-stranded conformation polymorphism and dideoxyfingerprinting. J Clin Microbiol 33: 1617-1623, 1995.

16. Hurley DM, Accili D, Stratakis CA, Karl M, Vamvakopoulos N, Rorer E, Constantine K, Taylor SI, Chrousos GP. Point mutation causing a single amino acid substitution in the hormone binding domain of the glucocorticoid receptor in familial glucocosticoid resistance. J Clin Invest 87: 680-686, 1991.

17. Ross J . Control of messenger RNA stability in higher eukaryotes. Trends Genet 12: 171- 175, 1996. 